\title{
Stress-assisted Grain Growth in Nanocrystalline Metals: Grain Boundary Mediated Mechanisms and Stabilization through Alloying
}

\author{
Yang Zhang ${ }^{1}$, Garritt J. Tucker ${ }^{2}$, and Jason R. Trelewicz ${ }^{1, *}$ \\ ${ }^{1}$ Department of Materials Science and Engineering, Stony Brook University, Stony Brook, NY 11794 \\ ${ }^{2}$ Department of Materials Science and Engineering, Drexel University, Philadelphia, PA 19104 \\ *Corresponding Author at Stony Brook University: jason.trelewicz@stonybrook.edu
}

\begin{abstract}
The mechanisms of stress-assisted grain growth are explored using molecular dynamics simulations of nanoindentation in nanocrystalline $\mathrm{Ni}$ and $\mathrm{Ni}-1$ at. $\% \mathrm{P}$ as a function of grain size and deformation temperature. Grain coalescence is primarily confined to the high stress region beneath the simulated indentation zone in nanocrystalline $\mathrm{Ni}$ with a grain size of $3 \mathrm{~nm}$. Grain orientation and atomic displacement vector mapping demonstrates that coalescence transpires through grain rotation and grain boundary migration, which are manifested in the grain interior and grain boundary components of the average microrotation. A doubling of the grain size to 6 $\mathrm{nm}$ and addition of 1 at.\% $\mathrm{P}$ eliminates stress-assisted grain growth in Ni. In the absence of grain coalescence, deformation is accommodated by grain boundary-mediated dislocation plasticity and thermally activated in pure nanocrystalline Ni. By adding solute to the Ni grain boundaries, the temperature-dependent deformation behavior observed in both the lattice and grain boundaries inverts, indicating that the individual processes of dislocation and grain boundary plasticity will exhibit different activity based on boundary chemistry and deformation temperature.
\end{abstract}

\section{Keywords}

Nanocrystalline metals, grain growth, grain boundaries, nanoindentation, molecular dynamics 


\section{Introduction}

Nanocrystalline metals have demonstrated many improvements in important technological and engineering properties such as high strength and increased wear resistance [1-5]. A key microstructural feature that underpins these performance improvements is the higher volume fraction of grain boundaries that inherently accompanies a reduction in grain size to the nanometer regime. By drastically increasing the volume fraction of grain boundaries, deformation physics shift to interface-mediated mechanisms involving interactions between dislocation plasticity, grain boundary sliding, and shear localization [6-10]. In fact, many recent studies have underscored the complexity in quantifying the interplay of different mechanisms in accommodating strain and accompanying microstructural evolution at the nanoscale [11-13]. Grain boundaries are thus central to the performance of nanocrystalline metals and must be comprehensively understood to design, model, and predict the behavior of this novel class of materials.

Grain boundaries constitute higher energy regions as compared with atoms occupying lattice sites within the interior of the grains in a polycrystalline metal. Specifically in nanocrystalline materials where the total grain boundary area is far greater than traditional microcrystalline counterparts, the interfacial free energy dominates the total energy of the system. A significant driving force thus exists to reduce the total grain boundary area [14], which transpires through grain growth accommodated by mechanisms such as grain rotation and grain boundary migration [15-18]. Historically, grain growth was studied as a consequence of instabilities that required a thermal energy component to overcome the inherent barrier for activating curvaturedriven grain boundary motion. However, recent findings support spontaneous microstructural evolution in nanocrystalline metals at temperature regimes often considered insufficient to initiate grain boundary migration and grain growth $[19,20]$. 
An interesting implication of the far-from-equilibrium state occupied by nanocrystalline metals that served as an impetus for this study is the formation of microstructural instabilities under mechanical loading (e.g., uniaxial loading [21-25], indentation [26, 27], and sliding contact [28, 29]) at low homologous temperatures including cryogenic conditions. In the pioneering work by Weertman et al. [26], a decrease in hardness was reported during microindentation creep experiments on nanocrystalline copper and attributed to rapid grain growth within the plastic zone beneath the indenter tip. Indentation-induced grain coarsening during indentation was found to be exaggerated at cryogenic temperatures [27], suggesting a stress-driven rather than diffusioncontrolled mechanism. Direct observation of deformation-induced grain growth was accomplished using in situ nanoindentation at room temperature, which ascribed grain coalescence in nanocrystalline aluminum to enhanced grain boundary mobility coupled with grain rotation $[18$, 30]. Mechanical grain coarsening was also found to influence the tensile properties of nanocrystalline aluminum at room temperature through microsample tensile testing [22]. A reduction in strength and enhanced ductility were correlated to discontinuous grain growth, which was later determined to be coupled to the applied stress by combining quantitative microstructural analysis with a continuum characterization of stress gradients [31].

The coupling between stress and grain boundary motion has been explored extensively through molecular dynamics (MD) simulations employing a range of structural models including bicrystal configurations [32-35] as well as equiaxed nanocrystalline structures [36-39]. While the former approach has produced geometrical frameworks to describe the coupling between grain boundary migration and shear deformation, the latter has proven particularly convenient for understanding the constraints imposed by the grain boundary network on stress-assisted grain boundary motion. For example, the presence of triple junctions was shown to inhibit grain 
boundary migration through an asymmetric pinning effect that resulted in non-linear grain boundary motion [36]. Cooperative grain boundary migration and sliding was also found to be driven by shear stress accumulation along the grain boundaries in nanocrystalline aluminum [40] while simulations in nanocrystalline palladium demonstrated grain boundary migration to be accompanied by grain rotation [37]. Simulations of surface indentation on nanocrystalline nickel were employed to study competing mechanisms during deformation-induced grain coalescence, and the results indicated the grain coarsening process was rate-dependent and accompanied by dislocation plasticity and twinning [41].

The need for improved microstructural stability in nanocrystalline metals has stimulated a myriad of efforts to probe the efficacy of stabilizing nanostructures through suppressing grain boundary mobility/migration. Theoretical/computational work demonstrated avenues for reducing interfacial energy through grain boundary segregation to quell the thermodynamic driving force for grain growth $[14,42-46]$. The notion of stabilizing nanocrystalline structures through grain boundary doping has been substantiated for a range of binary alloy systems at temperatures approaching $30 \%$ of their melting point [47-49]. Stabilization against mechanical grain growth has also been accomplished in nanocrystalline aluminum directly through the addition of grain boundary solute atoms [50] and indirectly through the presence of impurities [51] that effectively pin the boundaries against sliding and/or migration under stress. In the absence of stress-assisted grain growth, a transition to competing deformation mechanisms can be expected that will depend on the nanocrystalline grain size as well as extrinsic factors such as temperature and strain rate.

In this paper, indentation-induced grain growth is explored using large-scale MD simulations of model Ni and Ni-P systems. Stress-assisted grain growth is first characterized within the high stress region beneath the simulated indentation zone in nanocrystalline $\mathrm{Ni}$ with an 
average grain size of $3 \mathrm{~nm}$. Solute stabilization and grain size effects are probed through the addition of 1 at.\% $\mathrm{P}$ and doubling of the nanocrystalline grain size in pure Ni to $6 \mathrm{~nm}$, respectively. Mechanisms accommodating mechanical grain growth are then characterized via grain orientation and atomic displacement vector mapping. The manifestation of these mechanisms in the atomic microrotation is quantified to explicate average microrotation trends, which when combined with the dislocation fraction, provide a more complete signature of the underlying deformation mechanisms. Based on these results, the role of grain size, grain boundary doping, and deformation temperature in stress-assisted grain growth is discussed in the context of mechanistic crossovers to contribute new insights into the deformation behavior of nanocrystalline metals.

\section{Simulation Methods}

Simulations were conducted using the Large-scale Atomic/Molecular Massively Parallel Simulator (LAMMPS) platform. Three nanocrystalline structures were generated through Voronoi tessellation with a $50 \times 50 \times 50 \mathrm{~nm}$ total cell size that contained approximately 11 million atoms, which is illustrated in Figure 1 with atoms delineated for the grain interior and grain boundary regions. The samples included pure Ni with grain sizes of 3 and $6 \mathrm{~nm}$ and Ni-1 at.\% P with a grain size of $3 \mathrm{~nm}$, and are also shown in Figure 1 denoted Ni-3nm, Ni-6nm, and NiP-3nm, respectively. The embedded-atom method (EAM) potential developed by Sheng et al. [52] was used for NiP-3nm structure. While this potential incorporates Ni-Ni interactions that fit the singlecomponent data, it was developed specifically to capture short and medium range order in a Ni-P metallic glass. To better capture the long-range order in the Ni-6nm structure, the Ni EAM potential from Zhou et al. [53] was employed and also applied to the Ni-3nm structure for consistency across the pure Ni simulations. 
The NiP-3nm sample was first subjected to a parallelized Monte Carlo (MC) procedure developed by Sadigh et al. [54] to minimize the system energy with respect to solute distribution. A total of 500,000 MC steps was performed to produce an energy-minimized structure with the majority of the $\mathrm{P}$ atoms occupying grain boundary sites, in excellent agreement with experimental nanocrystalline Ni-P alloys [55]. An energy minimization procedure was then applied to all three structures to reach a final relative energy convergence of $10^{-8}$ and followed by a 1 ns simulated anneal to generate more realistic and relaxed interfaces. Annealing temperatures of $650 \mathrm{~K}$ and $1175 \mathrm{~K}$ were employed for the $3 \mathrm{~nm}$ and $6 \mathrm{~nm}$ grain size structures, respectively, with a constant cooling rate of $1 \mathrm{~K} \cdot \mathrm{s}^{-1}$; the lower annealing temperature was selected to avoid thermal coarsening of the $3 \mathrm{~nm}$ grain size structures.

Periodic boundary conditions were applied in all directions during structural relaxation, but removed from the z-direction post-relaxation to provide a free surface for nanoindentation. Structures were restrained during loading by a force wall applied to the lower $\mathrm{z}$ surface of the simulation cell, and ideal frictionless surface indentation was simulated using a moving artificial external potential following the procedures described in Ref. [41]. The radial force function, $F=$ $K(r-R)^{2}$, was employed to simulate a spherical indenter of radius $R=15 \mathrm{~nm}$ with $K=10 \mathrm{eV} / \AA^{3}$ $=1.6 \mu \mathrm{N} / \mathrm{nm}^{2}$ and $r$ the distance from each atom to the center of the indenter. Two temperatures of 10 and $300 \mathrm{~K}$ were employed with a constant velocity of $5 \mathrm{~m} / \mathrm{s}$ to a maximum indentation depth of $5 \mathrm{~nm}$, which corresponded to $1 / 10^{\text {th }}$ the total thickness of the simulation cell in the z-direction - a common constraint imposed on indentation experiments. The normal stresses in the $\mathrm{x}$ - and $\mathrm{y}-$ directions were relaxed to zero to allow lateral expansion/contraction during indentation, and an isobaric-isothermal (NPT) ensemble was used to control the temperature and pressure with a timestep of $2 \mathrm{fs}$. 
A number of analytical techniques were employed to characterize atomic and grain configurations to map nanostructural evolution as a function of indentation depth. The common neighbor analysis (CNA) method was used to index each atom according to its local atomic coordination where green atoms exhibit face-centered cubic (FCC) coordination, red atoms hexagonal close-packed (HCP) coordination (e.g. atoms commonly found in stacking faults), and grey atoms other coordination widely located in grain boundaries. From the CNA representation, the grain boundary network was outlined and used to determine grain size distributions under a spherical grain approximation. The process of grain coalescence was tracked using inverse pole figures where grain orientations were assigned on an atomistic basis using a next-nearest neighbor analysis as follows. For each atom, the full set of next-nearest neighbors was first indexed based on a unit vector established from one of its 12 nearest neighbors. For an FCC lattice, these nextnearest neighbors lie in the $\{100\}$ family of planes, and the (100) plane that exhibited the smallest angle with the $x-y$ plane of the simulation cell was selected from this set of planes. The normal to this particular (100) plane was then projected onto the crystallographic space defined by the [001], [101], and [111] directions to assign a specific crystallographic orientation to each atom. This procedure was repeated for all atoms within a given grain, which collectively represent the crystallographic orientation for that particular grain.

Dislocation slip was quantified as a function of indentation depth using a slip vector analysis [12] where each atom's slip vector, $\boldsymbol{s}^{\alpha}$, is calculated as:

$$
\boldsymbol{s}^{\alpha}=-\frac{1}{n_{s}} \sum_{\beta \neq \alpha}^{n}\left(\boldsymbol{x}^{\alpha \beta}-\boldsymbol{X}^{\alpha \beta}\right)
$$

where $n$ and $n_{s}$ are atom $\alpha$ 's total number of nearest neighbors and slipped nearest neighbors, respectively. The variables $\boldsymbol{x}^{\alpha \beta}$ and $\boldsymbol{X}^{\alpha \beta}$ represent the interatomic distance vector between atom 
$\alpha$ and neighbor $\beta$ in a deformed condition and reference state, respectively. Through comparison with the Burger's vector for dislocation slip in $\mathrm{Ni}$, the slip vector was employed to calculate the fraction of atoms contributing to dislocation plasticity also referred to as the dislocation fraction, $\rho$. For $\mathrm{Ni}$ with a lattice constant of $3.520 \AA$, the slip vector for partial and full slip is approximately 1.43 and $2.49 \AA$, respectively.

Microrotation was used as an atomic analysis metric as it captures the deformation history in the local neighborhood around an atom of interest, based on a defined reference state. Thus, as the atomic neighborhood undergoes deformation or straining, microrotation is able to capture that deformation. Deformation mechanisms studied previously using the microrotation metric include dislocation slip, grain boundary sliding, migration, atomic shuffling, and twinning [12]. Microrotation was calculated following the procedures described in Tucker et al. [34] and thus only summarized here. The rotation tensor, $\boldsymbol{R}$, was first calculated from the deformation gradient, $\boldsymbol{F}$, and the right stretch tensor, $\boldsymbol{U}$ :

$$
\boldsymbol{R}=\boldsymbol{F} \boldsymbol{U}^{-1}
$$

where $\boldsymbol{F}$ is estimated following procedures in Zimmerman et al. [56] and $\boldsymbol{U}^{-1}$ is the inverse of the right stretch tensor. The skew-symmetric component of the rotation tensor, $\boldsymbol{R}_{\text {skew }}$ was then ascertained from the rotation tensor:

$$
\boldsymbol{R}_{\text {skew }}=\frac{1}{2}\left(\boldsymbol{R}-\boldsymbol{R}^{T}\right)
$$

The microrotation vector, $\phi_{k}$, was finally determined from $\boldsymbol{R}_{\text {skew }}$ :

$$
\phi_{k}=-\frac{1}{2} \epsilon_{i j k}\left(\boldsymbol{R}_{\text {skew }}\right)_{i j}
$$


For visualization in deformation snapshots, atoms are colored according to their magnitude of microrotation while quantitative trends employ the microrotation per atom denoted herein the average microrotation. Local atomic displacements are also tracked and correlated to microrotation in deformation snapshots. The open source OVITO software [57] was used in the CNA and displacement vector visualizations whereas the slip vector, microrotation, and inverse pole figure calculations and mapping were produced using custom post-processing codes designed for execution in $\mathrm{C}$; all analysis codes are available from the authors upon request.

\section{Microstructural Evolution during Surface Indentation}

In this section, deformation-induced grain growth is quantified for the model nanocrystalline Ni and Ni-1 at.\% P systems. Grain size was determined from the grain volumes by tracking each grain during indentation using atomic coordination, and initial distributions following relaxation are depicted in Figure 2 for each sample. Under a spherical grain approximation, the mean grain size was calculated from the distribution of grain volumes and mapped as a function of indentation depth. Analysis was restricted to atoms within the high-stress region directly beneath the indenter, which was defined as the volume where the shear stress at the maximum indentation depth of $5 \mathrm{~nm}$ exceeded $10 \%$ of the mean contact pressure under Hertzian spherical contact. To facilitate a direct comparison between the different structures, grain size at each indentation depth was normalized by the mean grain size following relaxation. Normalized grain sizes are summarized in Figure 3 as a function of indentation depth with corresponding deformation snapshots shown for the maximum indentation depth at $300 \mathrm{~K}$.

The mean grain size of the Ni-3nm sample increased during indentation by almost $10 \%$ relative to the as-relaxed structure. From the deformation snapshot, grain growth was confined to the region directly beneath the indenter, which corresponded to the volume employed in the grain 
growth analysis; grain growth was completely absent outside of this high-stress region, thus confirming the role of stress in microstructural coarsening during indentation. A decrease in the deformation temperature to $10 \mathrm{~K}$ suppressed stress-assisted grain growth, indicating the underlying deformation mechanisms are thermally activated in pure nanocrystalline $\mathrm{Ni}$. A doubling of the grain size to $6 \mathrm{~nm}$ and the addition of 1 at. \% P segregated to the grain boundaries eliminated deformation-induced grain growth regardless of the deformation temperature. The surface indentation simulations conducted by Tucker and Foiles [41] on a comparable $6 \mathrm{~nm}$ grain size nanocrystalline Ni sample produced roughly a 3\% increase in grain size following indentation to the same maximum depth of $5 \mathrm{~nm}$. In light of the similar nanocrystalline structures and identical relaxation procedures, the subtle disparity in the extent of indentation-induced grain growth was attributed to the different EAM potential employed in the present study.

It was evident from the deformation snapshot for the $\mathrm{Ni}-3 \mathrm{~nm}$ structure that indentationinduced grain growth occurred solely in the high stress region beneath the indenter. While the Ni6nm and NiP-3nm structures were subjected to identical indentation stress fields, the local grain size within this region remained consistent with the surrounding undeformed material. To gain further insight into the coalescence process, a small section of material $5 \mathrm{~nm}$ thick was extracted from within the high stress region of the Ni-3nm structure as depicted in Figure 4a relative to the loading configuration, and is shown in Figure $4 \mathrm{~b}$ with three grains labeled grains 1 , 2, and 3 demarcated based on their orientations from the inverse pole figure. The deformation response of this section to the global surface indentation at $300 \mathrm{~K}$ is shown in Figures 5 (a-c) for 3, 4, and 5 $\mathrm{nm}$ indentation depths, respectively. All three grains remained present following indentation to 3 $\mathrm{nm}$, but with an increase to $4 \mathrm{~nm}$, grain 1 grew at the expense of grain 2 . The gradual change in orientation of grain 2 indicated it underwent a cooperative rotation, which was facilitated by grain 
boundary dislocation nucleation/emission as evidenced by the dislocation debris in the center of the grain and commensurate with experiments on nanocrystalline Pd [15]. While grain 3 remained at this indentation depth, the boundary between grains 1 and 3 migrated slightly toward the lower edge of the section. An increase to the maximum indentation depth of $5 \mathrm{~nm}$ resulted in coalescence of grain 3 with grain 1, thus producing a single large grain with the (111) orientation originally exhibited by grain 1 .

While grain coalesce also transpired during indentation at $10 \mathrm{~K}$ as evident in Figures 5(df), there are a number of noteworthy differences from the behavior at $300 \mathrm{~K}$ - particularly at depths greater than $3 \mathrm{~nm}$ that have mechanistic implications for stress-assisted grain growth. First, extended partial dislocations were apparent in the region originally occupied by grain 2 following indentation to $4 \mathrm{~nm}$. Dislocations that were originally apart of the low-angle grain boundary between grains 1 and 2 also remained in the interior of grain 1 after indentation to $5 \mathrm{~nm}$. Collectively, these observations are indicative of a reduced dislocation mobility at low temperatures and possibly the activation of GB deformation sources that facilitated grain rotation and coalescence. Second, while grain boundary and triple junction migration was detected between grains 1 and 3, its extent was limited at $10 \mathrm{~K}$ as evidenced by grain 3 remaining at the maximum indentation depth in Figure 5f. Given that the indentation-loading configuration was identical at both temperatures, the differences between 10 and $300 \mathrm{~K}$ can be attributed to the thermal activation component of the mechanisms accommodating mechanical grain growth explored in more detail in the remaining sections.

\section{Mechanisms Accommodating Stress-assisted Grain Growth}

The qualitative analysis from Figure 5 provided insight into the mechanisms that accommodate stress-assisted grain coalescence. The processes of grain rotation and grain 
boundary migration appeared to transpire simultaneously; however, this was inferred from changes in grain orientations during an isolated coalescence event involving only three grains. A global signature of these mechanisms is needed to understand their apparent temperature dependence and role of competing deformation mechanisms as a function of nanocrystalline grain size. In this section, the mechanisms inferred from the grain orientation mapping are first confirmed, and their manifestation through the calculation of average microrotation are then quantified in the high stress region shown in Figure 4.

Local atomic displacement mapping was employed to help differentiate between the mechanisms that facilitated the coalescence of grains 2 and 3 with grain 1 at $300 \mathrm{~K}$. The center of mass of grain 1 served as a reference point for computing atomic displacement vectors, and the resulting atomic displacement map is shown in Figure 6a with atoms colored according to their CNA value. Grain outlines represent the original configuration from Figure 4, and the magnitude and direction of the displacement are indicated by the length and direction of the associated arrows. Atoms within grain 1 exhibited only subtle reconfigurations that were concentrated near the grain boundaries, thus confirming the stability of grain 1 throughout the indentation process. Coordinated atomic displacements were apparent in grain 2 where atoms rotated around the center of grain 1, in turn facilitating a collective grain rotation process and growth of grain 1 at the expense of grain 2. Atoms in grain 3 instead exhibited uncoordinated motion that enabled the boundary between grains 1 and 3 to migrate toward the bottom of the section as implied by the orientation maps in Figures 5(a-c). Grain growth was therefore accommodated by a cooperative process involving grain rotation and grain boundary migration facilitated by local atomic reconfigurations. 
Atoms are recolored in Figure $6 \mathrm{~b}$ according to their atomic microrotation magnitude with the section divided into three regions along the grain boundaries emanating from the triple junction between the three delineated grains. In the region containing grain 1, atoms within grain interiors exhibited near-zero atomic microrotations while moderate microrotations were apparent for atoms located in adjacent grain boundaries. The regions containing grains 2 and 3 exhibited moderateto-high atomic microrotation values over the range of $0.5-1$, which derived from the atomic reconfigurations that facilitated growth of grain 1 via the rotation of grain 2, and migration of the boundary between grains 1 and 3. The average microrotation (i.e. microrotation per atom) was determined for each of the three regions during indentation at $300 \mathrm{~K}$ and plotted as a function of indentation depth in Figures $7 \mathrm{a}$ and $7 \mathrm{~b}$ for grain boundary and for grain interior (FCC) atoms, respectively. Not only were the qualitative observations from the atomic microrotation mapping in Figure $6 \mathrm{~b}$ confirmed, new insights into the atomic mechanisms enabling grain rotation and grain boundary migration were gained through collective analysis of the trends with indentation depth.

The increase in the average atomic microrotation of the grain boundaries commenced at different indentation depths of approximately 2 and $3 \mathrm{~nm}$ for grains 2 and 3, respectively, which can be attributed to a combination of factors such as the evolving stress state during indentation and the myriad of cooperative grain boundary deformation mechanisms such as shuffling/sliding [40], migration [20], and dislocation nucleation/absorption [58]. Although the abrupt increases in the average FCC microrotation also occurred at different indentation depths of 3 and $4 \mathrm{~nm}$ for grains 2 and 3, respectively, they consistently initiated at larger indentation depths relative to the average grain boundary microrotation. The primary implication is that atomic deformation or strain accommodation processes in the grain boundaries support both grain rotation and grain boundary migration under stress, which reoriented the lattice planes of grains 2 and 3 to align with 
the (111) orientation of grain 1. Atomic reconfigurations in the migrating grain boundary facilitated local reorientation of the lattice planes as the boundary swept through grain 3 . Conversely, a collective rotation of the atoms within grain 2 was enabled by atomic shuffling in the grain boundaries between this grain and all surrounding grains, thus leading to the most drastic increase in the grain boundary microrotation. It is finally important to note that grain boundary plasticity in the absence of mechanical grain growth also manifests as increased atomic microrotations [34]. Consequently, average atomic microrotation must be tracked collectively for grain boundary and grain interior mechanisms to serve as a global mechanistic signature of stressassisted grain growth in nanocrystalline metals.

\section{The Role of Grain Size, Grain Boundary Dopants, and Deformation Temperature}

Mechanical grain growth in nanocrystalline $\mathrm{Ni}$ was suppressed by a reduction in temperature and/or the addition of solute, which primarily segregated to the grain boundaries. A doubling of the grain size to $6 \mathrm{~nm}$ also eliminated grain coalescence under stress, thus implying that the activation of a different set of competing deformation mechanisms was necessary to accommodate plastic strain during indentation as a function of grain size. The grain boundary and FCC components of the average microrotation are studied in this section to investigate grain size, solute, and temperature effects, and compared with dislocation fractions to understand mechanistic transitions during stress-assisted grain growth. The average grain boundary and FCC microrotation from the high stress region were computed for each structure deformed at $300 \mathrm{~K}$ and plotted as a function of indentation depth in Figures $8 \mathrm{a}$ and $8 \mathrm{~b}$, respectively. The dislocation fractions were determined from the same simulations, and their scaling with indentation depth is shown in Figure 8c for each structure. The components of the average microrotation as well as 
the dislocation fractions increased monotonically with indentation depth for all the structures due to the expansion of the plastic zone under an evolving stress field.

For pure nanocrystalline $\mathrm{Ni}$, the average grain boundary microrotation was nearly identical for the Ni-3nm and Ni-6nm structures indicating that local grain boundary plasticity was active at both these nanocrystalline grain sizes despite the different volume fractions of grain boundaries in these microstructures. Conversely, the average atomic microrotation in FCC regions (i.e. grain interiors) of the Ni-6nm sample, which did not exhibit microstructural evolution, was suppressed relative to the Ni-3nm with appreciable stress-assisted grain growth. The increase in initial grain size thus inhibited the reorientation of lattice planes via grain rotation and grain boundary migration to facilitate grain coalescence under stress despite the prevalence of grain boundary deformation. In other words, the distribution of strain accommodation mechanisms within the microstructures is strongly tied to average grain size in pure Ni. Intragranular plasticity instead transpired through dislocation slip mediated by grain boundary plasticity as evidenced by the enhanced dislocation fraction in the Ni-6nm relative to the Ni-3nm structure. In the absence of stress-assisted grain growth, this mechanism represents a common deformation mode in nanocrystalline metals with grain sizes near the Hall-Petch breakdown [5].

The NiP-3nm structure with $\mathrm{P}$ atoms predominantly segregated to the grain boundaries exhibited a reduced grain boundary microrotation as compared with pure nanocrystalline Ni across all indentation depths. Nanocrystalline structures in Ni-P alloys have been demonstrated to be less susceptible to microstructural instabilities due to the $\mathrm{P}$ atoms augmenting the grain boundary energy [43]. The reduced grain boundary microrotation thus signaled a transition to a more stable interfacial configuration where the enrichment of $\mathrm{P}$ atoms limits local atomic reconfigurations in the grain boundaries under stress. Thus, due to $\mathrm{P}$ segregation to the grain boundaries in $\mathrm{Ni}$, the 
activation barrier for common grain boundary deformation processes effectively increases - owing to the stabilization of the boundaries. Collectively, the reduced microrotation for the grain interior and boundary regions indicated that the mechanisms facilitating stress-assisted grain growth were suppressed by the addition of $\mathrm{P}$, and strain was instead accommodated by enhanced dislocation slip (i.e. relative to the Ni-3nm structure) as apparent in Figure 8c.

The temperature effects were explored using normalized microrotation and dislocation factors, which were ascertained by dividing the average microrotations and dislocation fractions from the $300 \mathrm{~K}$ indentation simulations with the respective quantities from the identical simulations performed at $10 \mathrm{~K}$ as follows:

$$
\begin{gathered}
\lambda=\frac{\phi_{k}(T=300 K)}{\phi_{k}(T=10 K)} \\
\mu_{D I S L}=\frac{\rho(T=300 K)}{\rho(T=10 K)}
\end{gathered}
$$

In Eq. (3), $\lambda$ represents the normalized average microrotation factor and is calculated separately for the grain boundary $\left(\lambda_{G B}\right)$ and grain interior $\left(\lambda_{F C C}\right)$ regions, and $\mu_{D I S L}$ the normalized dislocation fraction. Thermally activated processes in this framework would exhibit microrotation and dislocation factors greater than unity while values less than unity indicate an inverse temperature dependence. The microrotation and dislocation factors are depicted in the semi-log plots shown in Figures 9(a-c) with the former separated for the grain boundary and grain interior regions akin to the microrotation analysis in Figure 8.

Grain boundary plasticity was thermally activated in pure nanocrystalline Ni regardless of grain size as indicated by $\lambda_{G B}$ exhibiting values greater than unity across all indentation depths. Grain boundary mechanisms were activated at low indentation strains and followed by dislocation 
slip mediated by the grain boundaries, which was demonstrated for nanocrystalline copper of similar grain size [11] and confirmed by the delayed onset of dislocation plasticity for Ni-3nm in Figure 8c. While dislocation plasticity was initially thermally activated (i.e. at shallow indentation depths), the temperature dependence diminished at larger indentation depths signaling a transition in the fundamental role of dislocation slip during strain accommodation. Finally, while $\lambda_{F C C}$ was consistently greater than unity for the Ni-3nm structure and indicative of a thermally activated deformation process transpiring in the grain interior regions, its convergence to unity in Ni-6nm signified that lattice deformation was independent of temperature at larger nanocrystalline grain sizes and indentation depths. The conflicting $\lambda_{F C C}$ trends can be attributed to the different intragranular deformation mechanisms operating at each grain size - grain rotation facilitating stress-assisted grain coalescence in Ni-3nm and dislocation slip in Ni-6nm, both of which were facilitated by grain boundary plasticity. Consequently, the process of stress-assisted grain coalescence was thermally activated, and an increase in the deformation temperature or decrease in grain size will accentuate mechanical grain growth in pure nanocrystalline $\mathrm{Ni}$.

The nanocrystalline Ni-P alloy exhibited starkly different behavior despite only a small amount of solute added to the structure, which segregated to the grain boundaries. The microrotation temperature factors $\lambda_{G B}$ and $\lambda_{F C C}$ were less than unity over all indentation depths indicating higher average atomic microrotation in those regions at lower temperatures - a fundamental shift in the competition of deformation mechanisms to accommodate microstructural strain as compared to pure Ni. These results suggest that the ability of the grain boundaries in the Ni-P system to undergo deformation at higher temperatures due to atomic shuffling and rearrangement (leading to migration, dislocation nucleation, and sliding) is significantly suppressed by alloying. Thus, the individual processes of grain boundary plasticity will exhibit 
different activity based on boundary chemistry and temperatures. By adding solute into the $\mathrm{Ni}$ grain boundaries, our results show that the temperature-dependent deformation behavior observed in both the lattice and grain boundaries inverts. This is both interesting and noteworthy as although the structure-property relationships of metallic grain boundaries are complex (dependent on structure, chemistry, temperature, loading, etc.) and still being fully understood, the 'inverse' or 'anti' thermal dependence observed in our Ni-P is seemingly similar to some individual grain boundary shear deformation behavior previously noted [59-61]. Further exploration of antithermal behavior is necessary to uncover the mechanisms responsible for this shift; however, to do so comprehensively is beyond the scope of the present work and will rely on a more complete mapping of individual grain boundaries and their connectivity to properly capture topological effects from the microstructure.

\section{Conclusions}

Molecular dynamics simulations of surface nanoindentation on nanocrystalline $\mathrm{Ni}$ with a grain size of $3 \mathrm{~nm}$ demonstrated that grain growth was stress-assisted during deformation. An increase in grain size and the addition of $\mathrm{P}$ segregated to the grain boundaries suppressed stressassisted grain growth in nanocrystalline metals. Grain orientation and atomic displacement vector mapping indicated that grain coalescence was facilitated by a cooperative process involving grain rotation and grain boundary migration. The manifestation of these processes in the average microrotation combined with dislocation fractions provided new insights into the dependence of stress-assisted grain growth on grain size, solute concentration, and temperature as follows:

i. For pure nanocrystalline Ni, the average grain boundary microrotation was independent of grain size indicating that local grain boundary plasticity was active despite the varying grain boundary volume fraction. Conversely, the average FCC microrotation 
in the Ni-6nm sample was markedly reduced relative to the Ni-3nm structure. Reorientation of lattice planes via grain rotation and grain boundary migration was thus inhibited at the larger nanocrystalline grain size and intragranular plasticity instead transpired through dislocation slip mediated by grain boundary plasticity.

ii. Grain boundary plasticity was thermally activated in pure nanocrystalline Ni and independent of grain size. Consequently, the process of stress-assisted grain growth and transition to grain boundary-mediated dislocation plasticity at larger nanocrystalline grain sizes were thermally activated in nanocrystalline $\mathrm{Ni}$.

iii. Temperature-dependent deformation behavior in both the lattice and grain boundaries inverted due to the addition of $\mathrm{P}$ atoms to the grain boundaries. This 'inverse' or 'anti' thermal dependence is commensurate with other findings on grain boundary shear deformation and underscores the significance of grain boundary chemistry in the mechanical stability of nanocrystalline metals.

Collective analysis of atomic microrotation, dislocation fraction, and their scaling with indentation depth and temperature provided a more complete signature of the mechanisms accommodating stress-assisted grain growth in nanocrystalline metals. Based on these results, a new fundamental shift in the competition of deformation mechanisms was identified that has important implications for the role of grain boundary-mediated plasticity in stress-coupled grain boundary migration and the deformation behavior of interface-dominated materials.

\section{Acknowledgements}

Support for this work was provided through the National Science Foundation under Grant No. DMR-1410941 for YZ and JRT, and DMR-1410970 for GJT. Results in this paper were 
obtained using the high-performance Handy and LIRED computing systems through the Institute for Advanced Computational Science at Stony Brook University. The authors also gratefully acknowledge the use of computing resources at the Center for Functional Nanomaterials, which is a US DOE Office of Science Facility at Brookhaven National Laboratory under Contract No. DESC0012704. 


\section{References}

[1] K.S. Kumar, H. Van Swygenhoven, S. Suresh. Mechanical behavior of nanocrystalline metals and alloys, Acta Materialia 51 (2003) 5743-5774.

[2] T.J. Rupert, W.J. Cai, C.A. Schuh. Abrasive wear response of nanocrystalline Ni-W alloys across the Hall-Petch breakdown, Wear 298 (2013) 120-126.

[3] J.R. Trelewicz, C.A. Schuh. The Hall-Petch breakdown at high strain rates: Optimizing nanocrystalline grain size for impact applications, Applied Physics Letters 93 (2008).

[4] M.A. Meyers, A. Mishra, D.J. Benson. Mechanical properties of nanocrystalline materials, Prog Mater Sci 51 (2006) 427-556.

[5] M. Dao, L. Lu, R.J. Asaro, J.T.M. De Hosson, E. Ma. Toward a quantitative understanding of mechanical behavior of nanocrystalline metals, Acta Materialia 55 (2007) 4041-4065.

[6] H. Van Swygenhoven, J.R. Weertman. Deformation in nanocrystalline metals, Mater Today 9 (2006) 24-31.

[7] J.R. Trelewicz, C.A. Schuh. The Hall-Petch breakdown in nanocrystalline metals: A crossover to glass-like deformation, Acta Materialia 55 (2007) 5948-5958.

[8] V. Yamakov, D. Wolf, S.R. Phillpot, A.K. Mukherjee, H. Gleiter. Deformation mechanism crossover and mechanical behaviour in nanocrystalline materials, Philosophical Magazine Letters 83 (2003) 385-393.

[9] Z.W. Shan, E.A. Stach, J.M.K. Wiezorek, J.A. Knapp, D.M. Follstaedt, S.X. Mao. Grain boundary-mediated plasticity in nanocrystalline nickel, Science 305 (2004) 654-657.

[10] T. Zhu, J. Li, A. Samanta, H.G. Kim, S. Suresh. Interfacial plasticity governs strain rate sensitivity and ductility in nanostructured metals, Proceedings of the National Academy of Sciences of the United States of America 104 (2007) 3031-3036.

[11] B. Cheng, J.R. Trelewicz. Mechanistic coupling of dislocation and shear transformation zone plasticity in crystalline-amorphous nanolaminates, Acta Materialia 117 (2016) 293-305.

[12] G.J. Tucker, S. Tiwari, J.A. Zimmerman, D.L. McDowell. Investigating the deformation of nanocrystalline copper with microscale kinematic metrics and molecular dynamics, Journal of the Mechanics and Physics of Solids 60 (2012) 471-486.

[13] T.J. Rupert. Strain localization in a nanocrystalline metal: Atomic mechanisms and the effect of testing conditions, Journal of Applied Physics 114 (2013) -.

[14] J. Weissmuller. Alloy effects in nanostructures, Nanostructured Materials 3 (1993) 261272.

[15] L. Wang, J. Teng, P. Liu, A. Hirata, E. Ma, Z. Zhang, M.W. Chen, X. Han. Grain rotation mediated by grain boundary dislocations in nanocrystalline platinum, Nature Communications 5 (2014).

[16] D. Farkas, S. Mohanty, J. Monk. Linear Grain Growth Kinetics and Rotation in Nanocrystalline Ni, Physical Review Letters 98 (2007) 165502.

[17] J. Kacher, I.M. Robertson, M. Nowell, J. Knapp, K. Hattar. Study of rapid grain boundary migration in a nanocrystalline $\mathrm{Ni}$ thin film, Materials Science and Engineering a-Structural Materials Properties Microstructure and Processing 528 (2011) 1628-1635.

[18] M. Legros, D.S. Gianola, K.J. Hemker. In situ TEM observations of fast grain-boundary motion in stressed nanocrystalline aluminum films, Acta Materialia 56 (2008) 3380-3393.

[19] M. Ames, J. Markmann, R. Karos, A. Michels, A. Tschope, R. Birringer. Unraveling the nature of room temperature grain growth in nanocrystalline materials, Acta Materialia 56 (2008) 4255-4266. 
[20] D. Farkas, A. Froseth, H. Van Swygenhoven. Grain boundary migration during room temperature deformation of nanocrystalline Ni, Scripta Materialia 55 (2006) 695-698.

[21] S. Brandstetter, K. Zhang, A. Escuadro, J.R. Weertman, H. Van Swygenhoven. Grain coarsening during compression of bulk nanocrystalline nickel and copper, Scripta Materialia 58 (2008) 61-64.

[22] D.S. Gianola, S. Van Petegem, M. Legros, S. Brandstetter, H. Van Swygenhoven, K.J. Hemker. Stress-assisted discontinuous grain growth and its effect on the deformation behavior of nanocrystalline aluminum thin films, Acta Materialia 54 (2006) 2253-2263.

[23] D.S. Gianola, D.H. Warner, J.F. Molinari, K.J. Hemker. Increased strain rate sensitivity due to stress-coupled grain growth in nanocrystalline Al, Scripta Materialia 55 (2006) 649-652.

[24] J.A. Sharon, P.C. Su, F.B. Prinz, K.J. Hemker. Stress-driven grain growth in nanocrystalline Pt thin films, Scripta Materialia 64 (2011) 25-28.

[25] G.J. Fan, L.F. Fu, H. Choo, P.K. Liaw, N.D. Browning. Uniaxial tensile plastic deformation and grain growth of bulk nanocrystalline alloys, Acta Materialia 54 (2006) 4781-4792.

[26] K. Zhang, J.R. Weertman, J.A. Eastman. The influence of time, temperature, and grain size on indentation creep in high-purity nanocrystalline and ultrafine grain copper, Applied Physics Letters 85 (2004) 5197-5199.

[27] K. Zhang, J.R. Weertman, J.A. Eastman. Rapid stress-driven grain coarsening in nanocrystalline $\mathrm{Cu}$ at ambient and cryogenic temperatures, Applied Physics Letters 87 (2005) 061921.

[28] T.J. Rupert, C.A. Schuh. Sliding wear of nanocrystalline Ni-W: Structural evolution and the apparent breakdown of Archard scaling, Acta Materialia 58 (2010) 4137-4148.

[29] N. Argibay, T. Furnish, B. Boyce, B. Clark, M. Chandross. Stress-dependent grain size evolution of nanocrystalline $\mathrm{Ni}-\mathrm{W}$ and its impact on friction behavior, Scripta Materialia 123 (2016) 26-29.

[30] M. Jin, A.M. Minor, E.A. Stach, J.W. Morris Jr. Direct observation of deformation-induced grain growth during the nanoindentation of ultrafine-grained Al at room temperature, Acta Materialia 52 (2004) 5381-5387.

[31] T.J. Rupert, D.S. Gianola, Y. Gan, K.J. Hemker. Experimental Observations of StressDriven Grain Boundary Migration, Science 326 (2009) 1686-1690.

[32] J.W. Cahn, Y. Mishin, A. Suzuki. Coupling grain boundary motion to shear deformation, Acta Materialia 54 (2006) 4953-4975.

[33] D. Caillard, F. Mompiou, M. Legros. Grain-boundary shear-migration coupling. II. Geometrical model for general boundaries, Acta Materialia 57 (2009) 2390-2402.

[34] G.J. Tucker, J.A. Zimmerman, D.L. McDowell. Shear deformation kinematics of bicrystalline grain boundaries in atomistic simulations, Model Simul Mater Sc 18 (2010).

[35] F. Mompiou, M. Legros, D. Caillard. SMIG model: A new geometrical model to quantify grain boundary-based plasticity, Acta Materialia 58 (2010) 3676-3689.

[36] M. Aramfard, C. Deng. Influences of triple junctions on stress-assisted grain boundary motion in nanocrystalline materials, Model Simul Mater Sc 22 (2014).

[37] A.J. Haslam, D. Moldovan, V. Yamakov, D. Wolf, S.R. Phillpot, H. Gleiter. Stressenhanced grain growth in a nanocrystalline material by molecular-dynamics simulation, Acta Materialia 51 (2003) 2097-2112.

[38] A. Hasnaoui, H. Van Swygenhoven, P.M. Derlet. Cooperative processes during plastic deformation in nanocrystalline fcc metals: A molecular dynamics simulation, Physical Review B 66 (2002) -. 
[39] M. Velasco, H. Van Swygenhoven, C. Brandl. Coupled grain boundary motion in a nanocrystalline grain boundary network, Scripta Materialia 65 (2011) 151-154.

[40] D.S. Gianola, D. Farkas, M. Gamarra, M.-r. He. The role of confinement on stress-driven grain boundary motion in nanocrystalline aluminum thin films, Journal of Applied Physics 112 (2012) 124313.

[41] G.J. Tucker, S.M. Foiles. Molecular dynamics simulations of rate-dependent grain growth during the surface indentation of nanocrystalline nickel, Materials Science and Engineering aStructural Materials Properties Microstructure and Processing 571 (2013) 207-214.

[42] J.R. Trelewicz, C.A. Schuh. Grain boundary segregation and thermodynamically stable binary nanocrystalline alloys, Physical Review B 79 (2009).

[43] F. Liu, R. Kirchheim. Nano-scale grain growth inhibited by reducing grain boundary energy through solute segregation, J Cryst Growth 264 (2004) 385-391.

[44] P.C. Millett, R.P. Selvam, A. Saxena. Stabilizing nanocrystalline materials with dopants, Acta Materialia 55 (2007) 2329-2336.

[45] F. Abdeljawad, S.M. Foiles. Stabilization of nanocrystalline alloys via grain boundary segregation: A diffuse interface model, Acta Materialia 101 (2015) 159-171.

[46] K.A. Darling, M.A. Tschopp, B.K. VanLeeuwen, M.A. Atwater, Z.K. Liu. Mitigating grain growth in binary nanocrystalline alloys through solute selection based on thermodynamic stability maps, Computational Materials Science 84 (2014) 255-266.

[47] T. Chookajorn, H.A. Murdoch, C.A. Schuh. Design of Stable Nanocrystalline Alloys, Science 337 (2012) 951-954.

[48] K.A. Darling, A.J. Roberts, Y. Mishin, S.N. Mathaudhu, L.J. Kecskes. Grain size stabilization of nanocrystalline copper at high temperatures by alloying with tantalum, Journal of Alloys and Compounds 573 (2013) 142-150.

[49] B.G. Clark, K. Hattar, M.T. Marshall, T. Chookajorn, B.L. Boyce, C.A. Schuh. Thermal Stability Comparison of Nanocrystalline Fe-Based Binary Alloy Pairs, JOM 68 (2016) 1625-1633. [50] R.I. Babicheva, S.V. Dmitriev, L.C. Bai, Y. Zhang, S.W. Kok, G.Z. Kang, K. Zhou. Effect of grain boundary segregation on the deformation mechanisms and mechanical properties of nanocrystalline binary aluminum alloys, Computational Materials Science 117 (2016) 445-454.

[51] M.R. He, S.K. Samudrala, G. Kim, P.J. Felfer, A.J. Breen, J.M. Cairney, D.S. Gianola. Linking stress-driven microstructural evolution in nanocrystalline aluminium with grain boundary doping of oxygen, Nature Communications 7 (2016).

[52] H.W. Sheng, E. Ma, M.J. Kramer. Relating Dynamic Properties to Atomic Structure in Metallic Glasses, Jom 64 (2012) 856-881.

[53] X.W. Zhou, R.A. Johnson, H.N.G. Wadley. Misfit-energy-increasing dislocations in vapor-deposited CoFe/NiFe multilayers, Physical Review B 69 (2004).

[54] Sadigh.Babak, Erhart.Paul, Stukowski.Alexander, Caro.Alfredo, Martinez.Enrique, Zepeda-Ruiz.Luis. Scalable parallel Monte Carlo algorithm for atomistic simulations of precipitation in alloys, Physical Review B 85 (2012).

[55] B. Farber, E. Cadel, A. Menand, G. Schmitz, R. Kirchheim. Phosphorus segregation in nanocrystalline Ni-3.6 at.\% $\mathrm{P}$ alloy investigated with the tomographic atom probe (TAP), Acta Materialia 48 (2000) 789-796.

[56] J.A. Zimmerman, D.J. Bammann, H.J. Gao. Deformation gradients for continuum mechanical analysis of atomistic simulations, International Journal of Solids and Structures 46 (2009) 238-253. 
[57] A. Stukowski. Visualization and analysis of atomistic simulation data with OVITO-the Open Visualization Tool, Modelling and Simulation in Materials Science and Engineering 18 (2010) 015012.

[58] H. Van Swygenhoven, P.M. Derlet, A.G. Froseth. Nucleation and propagation of dislocations in nanocrystalline fcc metals, Acta Materialia 54 (2006) 1975-1983.

[59] C.J. O'Brien, S.M. Foiles. Exploration of the mechanisms of temperature-dependent grain boundary mobility: search for the common origin of ultrafast grain boundary motion, Journal of Materials Science 51 (2016) 6607-6623.

[60] P.R. Cantwell, E.A. Holm, M.P. Harmer, M.J. Hoffmann. Anti-thermal behavior of materials, Scripta Materialia 103 (2015) 1-5.

[61] E.R. Homer, E.A. Holm, S.M. Foiles, D.L. Olmsted. Trends in Grain Boundary Mobility: Survey of Motion Mechanisms, Jom 66 (2014) 114-120. 


\section{Figures}

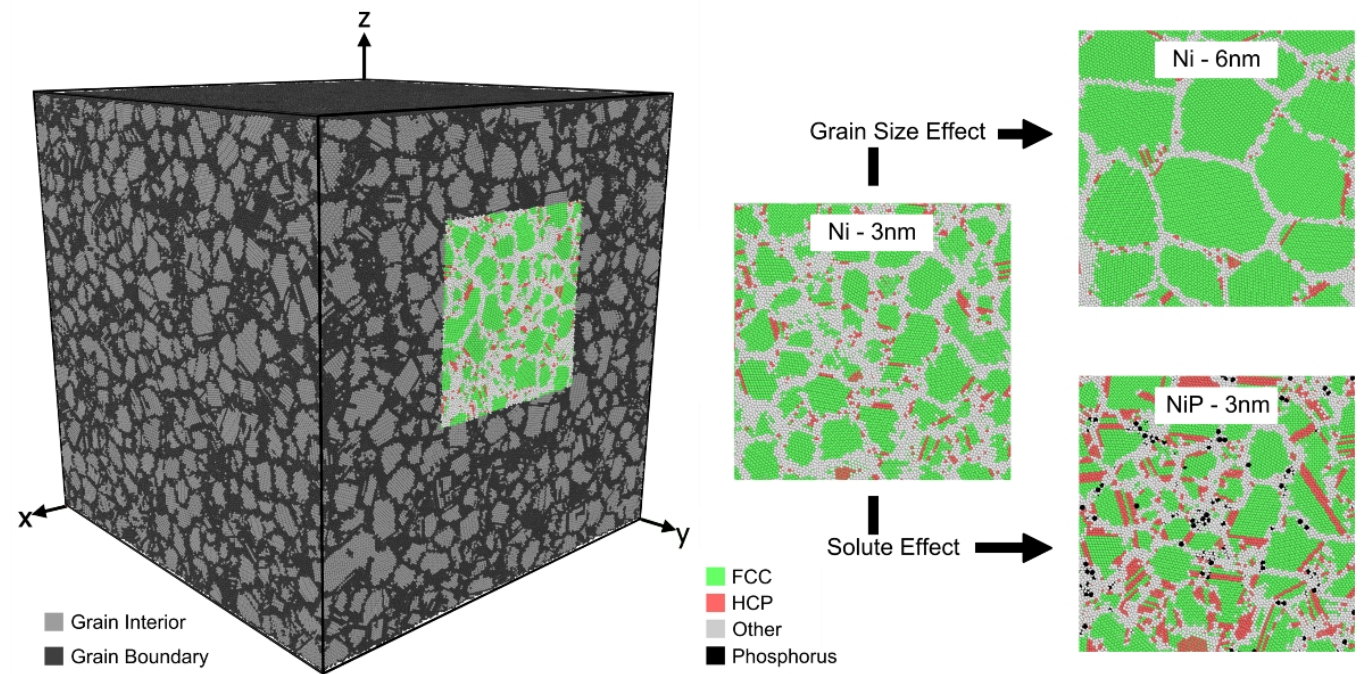

Figure 1: Overview of the $50 \times 50 \times 50 \mathrm{~nm}$ nanocrystalline simulation cell containing approximately 11 million atoms colored according to location within the grain structure in the $3 \mathrm{D}$ view. Small 2D sections with atoms colored according to the their CNA value are extracted from the $\mathrm{x}-\mathrm{z}$ plane and illustrated for the Ni-3nm structure with grain size and solute effects mapped via the Ni-6nm and NiP-3nm structures, respectively. The reader is referred to the web version of this article for interpretation of references to color. 


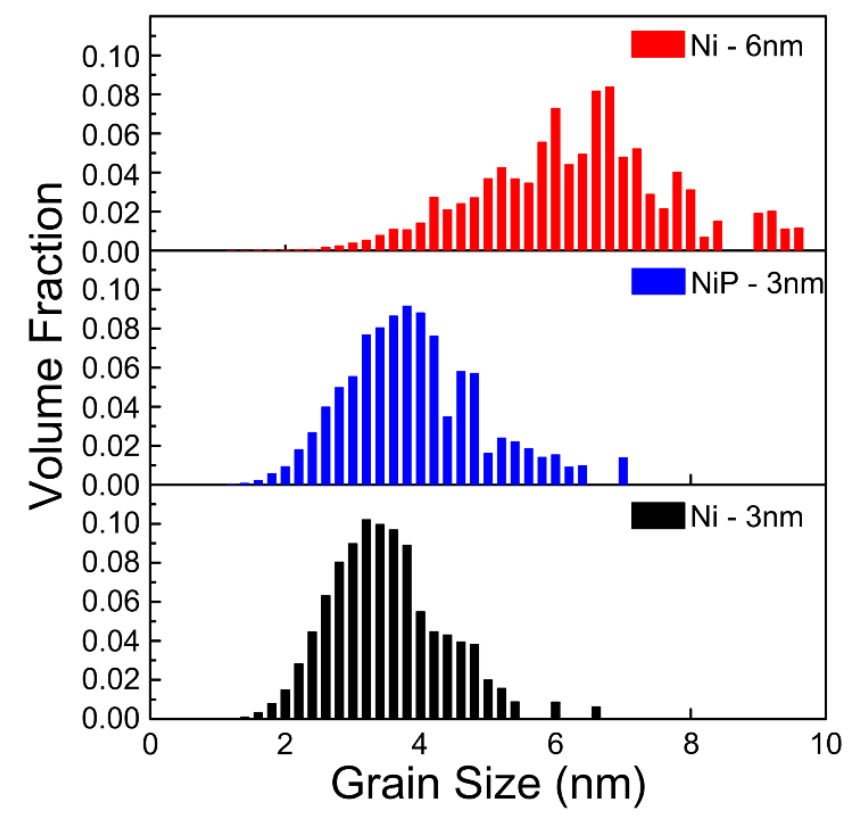

Figure 2: Grain size distributions for the Ni-3nm, NiP-3nm, and Ni-6nm structures where an increase in the mean grain size is evident for the latter relative to the $3 \mathrm{~nm}$ grain size structures. 


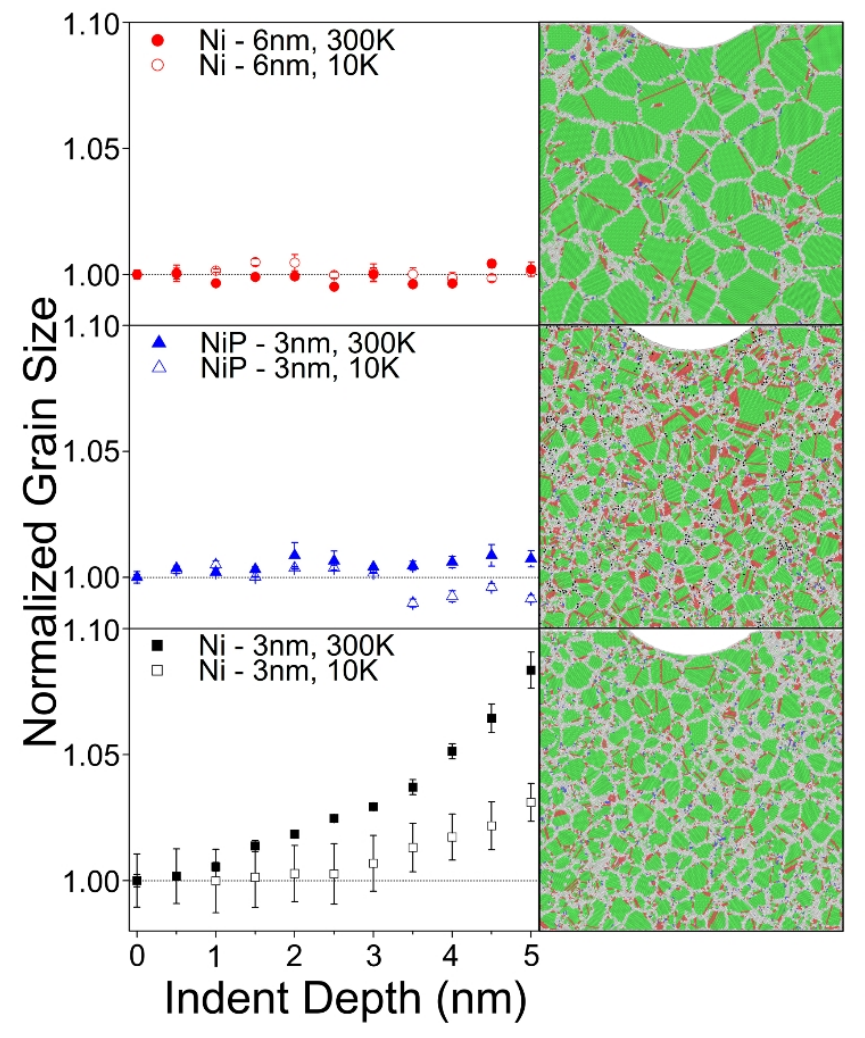

Figure 3: Normalized grain size as a function of indentation depth for the Ni-3nm, NiP$3 \mathrm{~nm}$, and Ni-6nm structures indented at 10 and $300 \mathrm{~K}$. Corresponding deformation snapshots are illustrated for the maximum indentation depth of $5 \mathrm{~nm}$ at $300 \mathrm{~K}$, and demonstrate that indentation-induced grain growth is limited to the high stress region of the Ni-3nm sample. 
a)

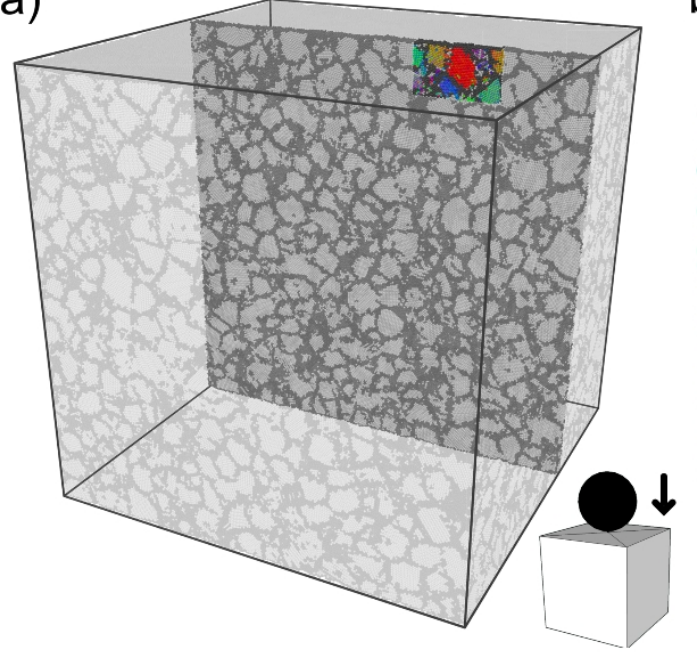

b)

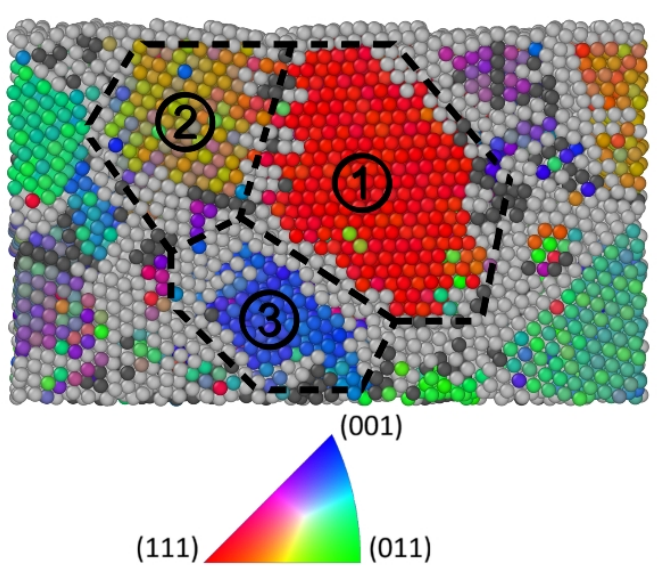

Figure 4: (a) Location of the $5 \mathrm{~nm}$-thick section for grain structure tracking within the high-stress region of the Ni-3nm structure. (b) Snapshot of the section prior to indentation containing three grains labeled 1,2, and 3 demarcated based on their crystallographic orientations from the inverse pole figure with grain boundaries denoted by the grey atoms. The reader is referred to the web version of this article for interpretation of references to color. 
a)

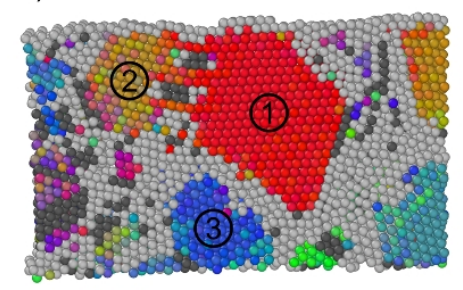

d)

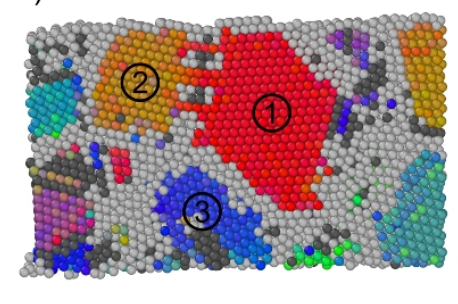

b)

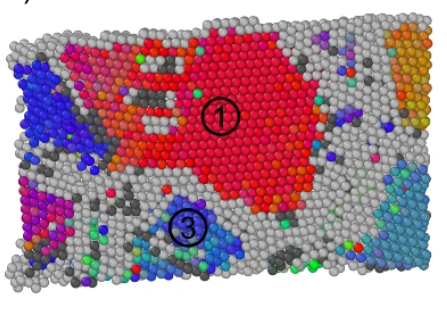

e)

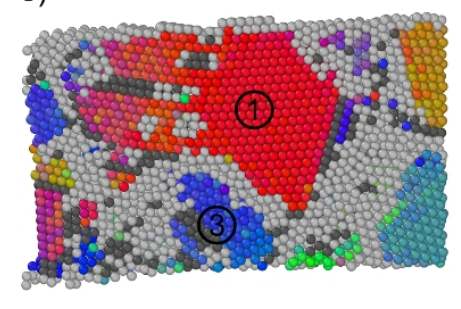

c)

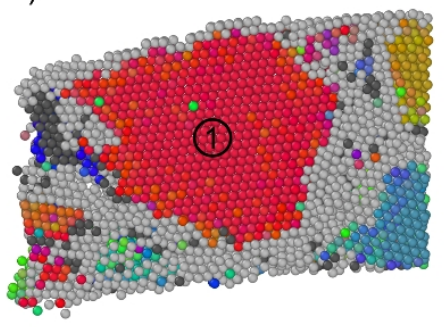

f)

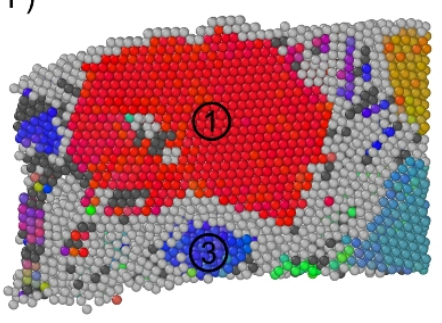

Figure 5: Tracking grain coalescence at indentation depths of 3, 4, and $5 \mathrm{~nm}$ (from leftto-right) and temperatures of $300 \mathrm{~K}$ (a-c) and $10 \mathrm{~K}(\mathrm{~d}-\mathrm{f})$. At $300 \mathrm{~K}$, grains 2 and 3 coalesced with grain 1 whereas grain 3 remained following indentation to $5 \mathrm{~nm}$ at $10 \mathrm{~K}$ in (f). Atoms are colored according to the inverse pole figure in Figure 4 with grain boundaries denoted by the grey atoms. The reader is referred to the web version of this article for interpretation of references to color. 
a)

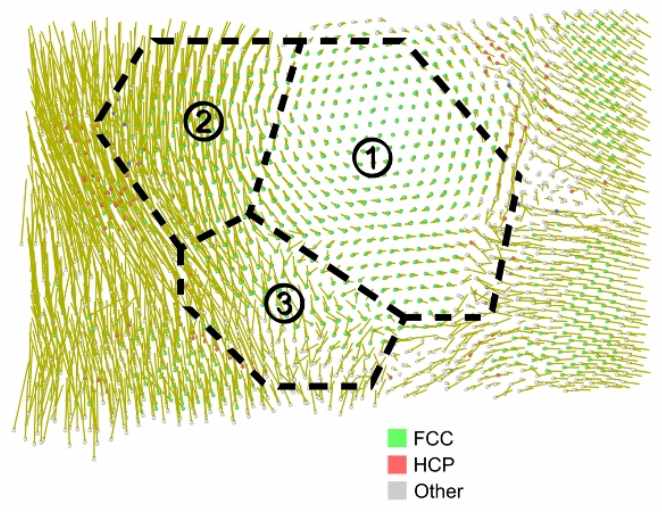

b)

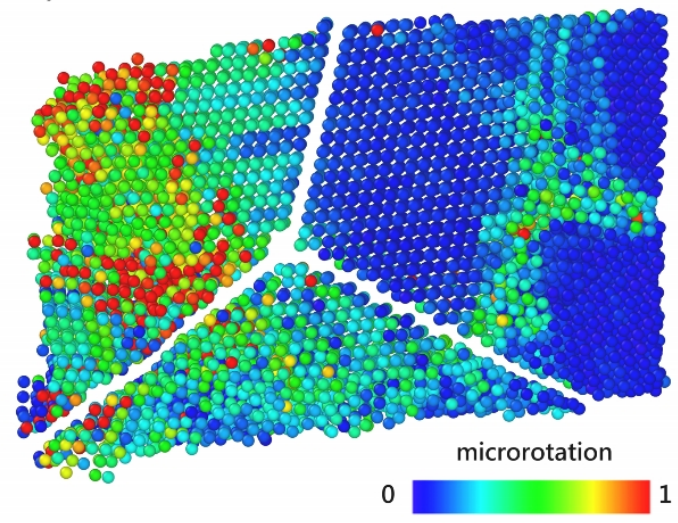

Figure 6: Snapshot of the section from the Ni-3nm structure at an indentation depth of 5 $\mathrm{nm}$ and temperature of $300 \mathrm{~K}$. (a) Mapping of atomic displacement vectors with the center of mass of grain 1 serving as a reference point and atoms colored by CNA value. (b) Division of the section along the grain boundaries that meet at the triple junction between grains 1,2, and 3 on the original grain overlay illustrated in (a) with atoms colored according to atomic microrotation value. The reader is referred to the web version of this article for interpretation of references to color. 
a)

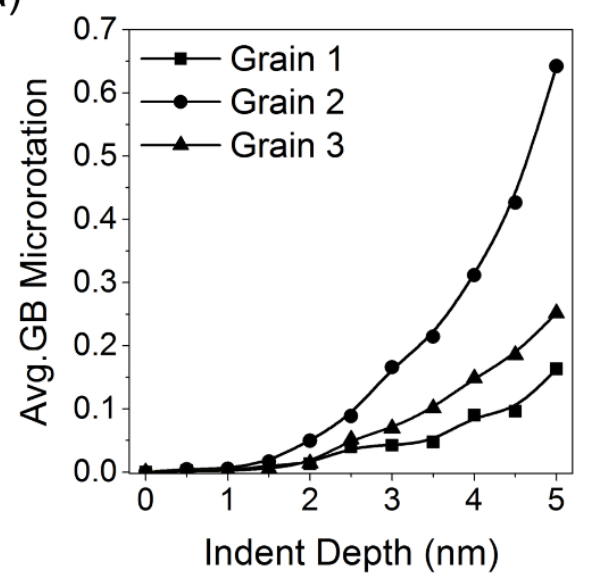

b)

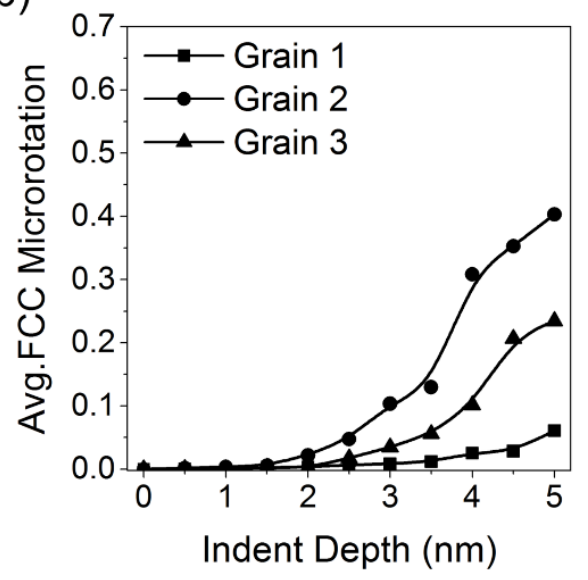

Figure 7: Average microrotation as a function of indentation depth for (a) grain boundary and (b) grain interior FCC atoms delineated for the regions corresponding to grains 1, 2, and 3 divided along the grain boundaries separating these grains as shown in Figure 6b. 

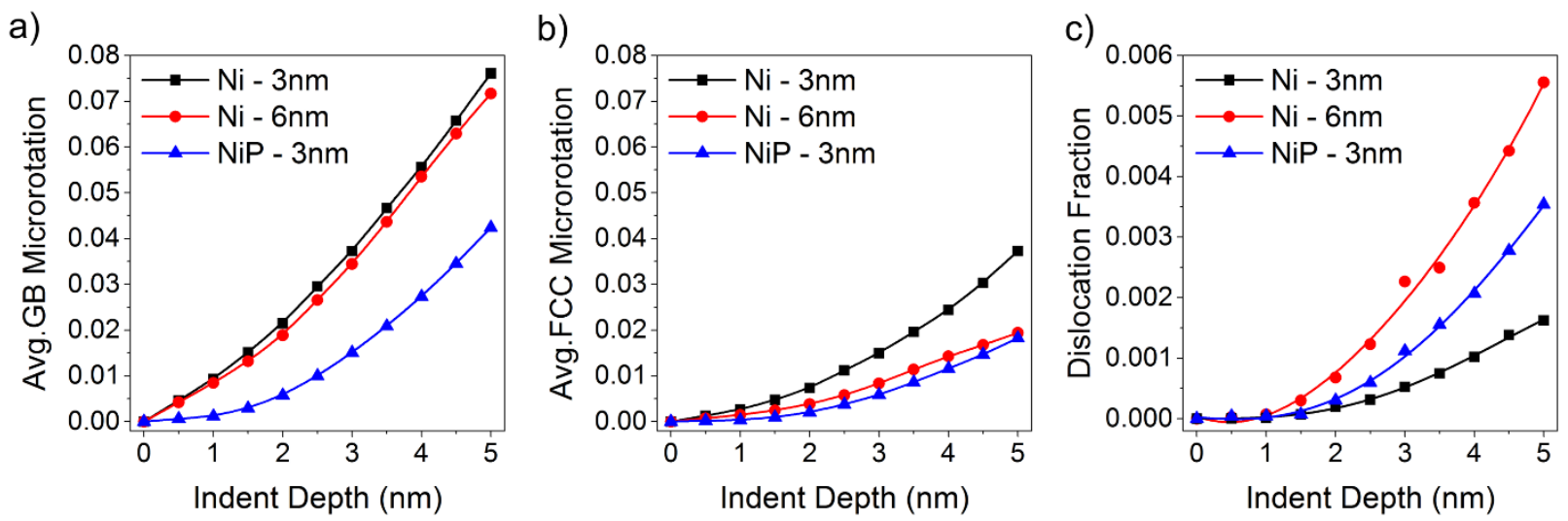

Figure 8: Global deformation metric trends with indentation depth at $300 \mathrm{~K}$ for (a) average grain boundary microrotation, (b) average grain interior FCC microrotation, and (c) dislocation fraction delineated for the Ni-3nm, Ni-6nm, and NiP-3nm structures. 
a)

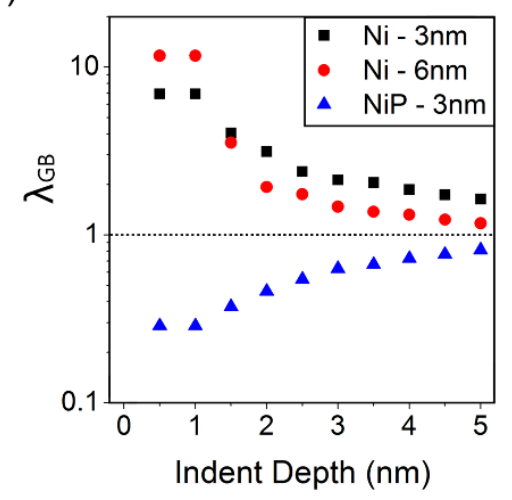

b)

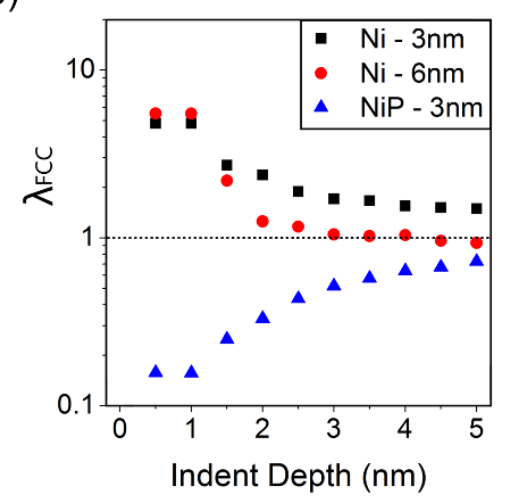

c)

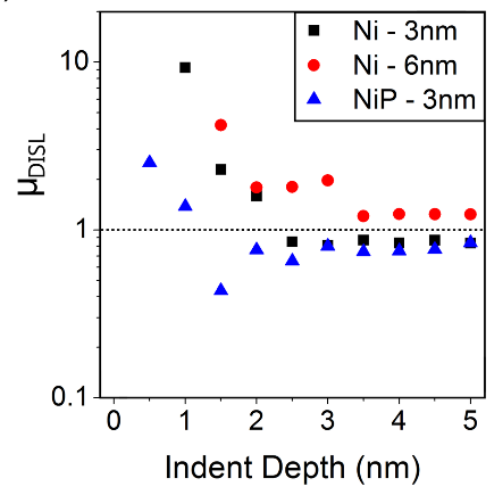

Figure 9: Global temperature dependence of the deformation metrics trends with indentation depth as captured by the normalized average microrotation factor in the (a) grain boundary and (b) grain interior regions, and (c) normalized dislocation fraction, delineated for the Ni-3nm, Ni-6nm, and NiP-3nm structures. 